\title{
A Competitive Advantage by Neonatally Engrafted Human Glial Progenitors Yields Mice Whose Brains Are Chimeric for Human Glia
}

\author{
Martha S. Windrem, ${ }^{1}$ Steven J. Schanz, ${ }^{1}$ Carolyn Morrow, ${ }^{1}$ Jared Munir, ${ }^{1}$ Devin Chandler-Militello, ${ }^{1}$ Su Wang, ${ }^{1}$ \\ and Steven A. Goldman ${ }^{1,2}$ \\ ${ }^{1}$ Center for Translational Neuromedicine, University of Rochester Medical Center, Rochester, New York 14642, and ${ }^{2}$ Center for Basic and Translational \\ Neuroscience, University of Copenhagen Faculty of Medicine, 2200 Copenhagen N, Denmark
}

\begin{abstract}
Neonatally transplanted human glial progenitor cells (hGPCs) densely engraft and myelinate the hypomyelinated shiverer mouse. We found that, in hGPC-xenografted mice, the human donor cells continue to expand throughout the forebrain, systematically replacing the host murine glia. The differentiation of the donor cells is influenced by the host environment, such that more donor cells differentiated as oligodendrocytes in the hypomyelinated shiverer brain than in myelin wild-types, in which hGPCs were more likely to remain as progenitors. Yet in each recipient, both the number and relative proportion of mouse GPCs fell as a function of time, concomitant with the mitotic expansion and spread of donor hGPCs. By a year after neonatal xenograft, the forebrain GPC populations of implanted mice were largely, and often entirely, of human origin. Thus, neonatally implanted hGPCs outcompeted and ultimately replaced the host population of mouse GPCs, ultimately generating mice with a humanized glial progenitor population. These human glial chimeric mice should permit us to define the specific contributions of glia to a broad variety of neurological disorders, using human cells in vivo.
\end{abstract}

Key words: cell transplant; chimera; demyelinating disease; glial progenitor; neural stem cell; oligodendrocytic progenitor

\section{Introduction}

In an effort to develop human cellular vectors for therapeutic remyelination, we have developed efficient methods by which to identify and isolate human glial progenitor cells (hGPCs), in quantities and purities appropriate for transplantation (Goldman et al., 2012). Using immune-deficient mice as hosts, we established a neonatal multisite delivery procedure that results in widespread hGPC engraftment throughout the brain and spinal cord, with infiltration of the forebrain, brainstem, and cerebellum, and ultimately the spinal cord and roots (Windrem et al., 2008). When delivered to myelin-deficient shiverer mice $\left(\mathrm{MBP}^{\text {shishi }}\right)$, these donor hGPCs, whether isolated from tissue (Windrem et al., 2004, 2008) or generated from human embryonic stem cells or induced pluripotential stem cells (Wang et al., 2013), exhibited efficient oligodendrocyte differentiation and myelination, as well as fibrous as-

Received April 13, 2014; revised Sept. 24, 2014; accepted 0ct. 18, 2014.

Author contributions: M.S.W., S.W., and S.A.G. designed research; M.S.W., S.J.S., C.M., J.M., D.C.-M., and S.W. performed research; M.S.W., S.J.S., C.M., J.M., and S.A.G. analyzed data; M.S.W. and S.A.G. wrote the paper.

This work was supported by the Mathers Charitable Foundation, National Institute of Neurological Disorders and Stroke R01NS75345, the National Institute of Mental Health Grants R01MH099578-01 and R01MH104701, the National Multiple Sclerosis Society, the Adelson Medical Research Foundation, the New York Stem Cell Research Program, and the Novo Nordisk Fondation.

S.A.G. and M.S.W. have a patent on chimeric mouse models (U.S. patent no. US7524491B2); the patent is owned by the University of Rochester, and the authors receive no income from it. The remaining authors declare no competing financial interests.

Correspondence should be addressed to either Dr. Martha Windrem or Dr. Steven Goldman, Center for Translational Neuromedicine, University of Rochester Medical Center, 601 Elmwood Avenue, Rochester, NY 14642. E-mail: martha_windrem@urmc.rochester.edu or steven_goldman@urmc.rochester.edu.

DOI:10.1523/JNEUROSCI.1510-14.2014

Copyright $\odot 2014$ the authors $\quad 0270-6474 / 14 / 3416153-09 \$ 15.00 / 0$ trocyte production, permitting the clinical rescue of these otherwise lethally hypomyelinated mice.

Yet in contrast to their bilineal oligodendrocytic and astrocytic fate competence in a hypomyelinated host, these xenografted hGPCs either remained as progenitors or differentiated into astrocytes in wild-type mice, revealing little oligodendrocytic differentiation, and thus suggesting the context dependence of their fate choice (Goldman et al., 2008). As a result, when hGPCs were xenografted into immunodeficient but otherwise wild-type neonatal mice, the recipient brains were effectively colonized by hGPCs and their derived astroglia (Han et al., 2013). Indeed, the resultant occupation of these brains by human glia was so robust that it prompted us to investigate the functional and behavioral consequences of this interspecific chimerization. We found that the glial chimeric mice exhibited both increased synaptic plasticity and improved cognitive performance, manifested by both enhanced long-term potentiation and improved performance in a variety of learning tasks (Han et al., 2013). In the context of that study, we were surprised to note that the forebrains of these animals were often composed primarily of human glia and their progenitors, with overt diminution in the relative proportion of resident mouse glial cells.

On the basis of these observations, we asked here whether neonatal human glial chimerization can yield the large-scale replacement of resident murine glial progenitor cells by hGPCs, whether this process can result in the effective humanization of the adult mouse with respect to its glial phenotypes, and if so, by what kinetics this process proceeds, and with what context- 
Table 1. Antibodies and dilutions used for histological analysis in this study

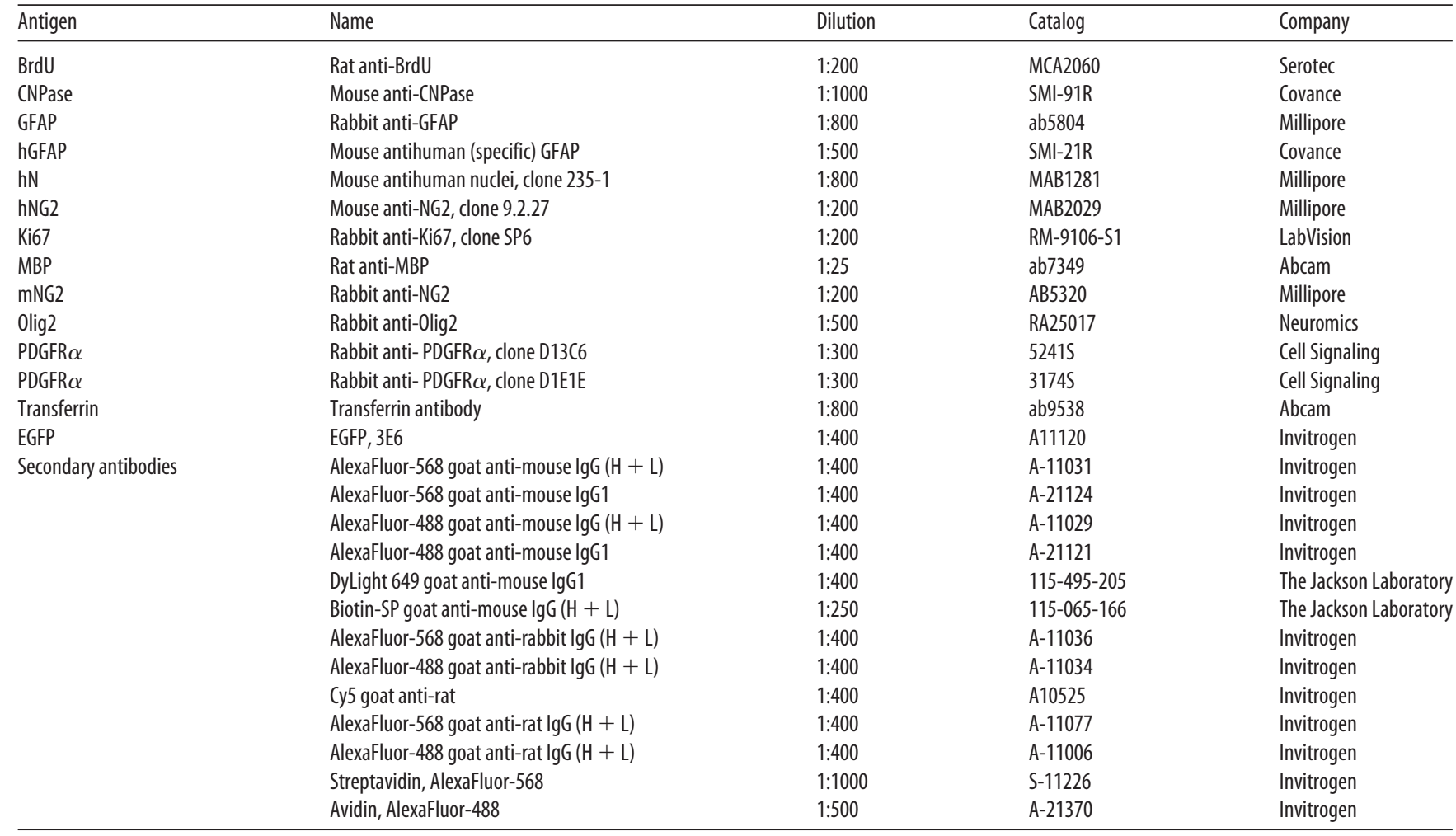

dependent determination of cell lineage and fate. In doing so, we have found that hGPCs exhibit a competitive dominance when xenografted into the mouse brain that results in the effective, and often complete, replacement of mouse glial progenitors by their human counterparts, with subsequent astrocytic differentiation, thereby yielding murine brains in which human glial cells predominate.

\section{Materials and Methods}

Human and mouse cell dissociation. For xenograft of human fetal GPCs, cells were extracted from second-trimester human fetuses (18-22 weeks gestation age) obtained at abortion. The forebrain ventricular/subventricular zones were dissected from the brain, the samples chilled on ice, minced and dissociated using papain/DNase, as described previously (Roy et al., 1999, 2000), always within $3 \mathrm{~h}$ of extraction. The dissociates were maintained overnight in minimal media of DMEM/F12/N1 with 10 $\mathrm{ng} / \mathrm{ml}$ bFGF. Samples were deidentified and obtained with the approval of the University of Rochester Research Subjects Review Board.

As controls, allografted mouse cells were obtained from $\mathrm{Tg}(\mathrm{CAG}$ EGFP)B5Nagy/J mice (The Jackson Laboratory). P1 pups were cryoanesthetized, their forebrains removed, and dissociated as above; like their human counterparts, the mouse cells were also maintained overnight in DMEM/F12/N1 with $10 \mathrm{ng} / \mathrm{ml}$ bFGF before sorting.

Sorting. Glial progenitor cells were isolated the day after tissue dissociation, using immunomagnetic sorting (MACS, Miltenyi Biotec), as described previously (Windrem et al., 2008). The human cells were incubated with mouse anti-PSA-NCAM (clone 2-2B, Millipore; clone $5 \mathrm{~A} 5$, DSHB), then washed and labeled with microbead-tagged rat antimouse IgM (Miltenyi), and the PSA-NCAM ${ }^{+}$cells removed by MACS depletion. The PSA-NCAM-depleted remainder was then incubated with $\mathrm{mAb}$ A2B5 supernatant (clone 105; ATCC) for $20 \mathrm{~min}$, then washed and tagged with microbead-tagged rat anti-mouse IgM (Miltenyi), and the A2B5 ${ }^{+}$cells separated by MACS selection. The bound cells were then eluted, yielding a highly enriched population of PSA-NCAM ${ }^{-} / \mathrm{A}_{2} \mathrm{~B} 5^{+}$ cells. After sorting, the cells were either maintained in vitro up to 2 weeks in DMEM/F12/N1 with $10 \mathrm{ng} / \mathrm{ml} \mathrm{bFGF}$ and $20 \mathrm{ng} / \mathrm{ml}$ PDGF-AA, or frozen and stored in liquid $\mathrm{N}_{2}$ at $2 \times 10^{6}$ cells $/ \mathrm{ml}$ in $7.5 \% \mathrm{DMSO} / 50 \%$ media (DMEM/F12/N1)/42.5\% ProFreeze-CDM (Lonza).

Mouse cells were incubated with mAb A2B5 supernatant for $20 \mathrm{~min}$, then washed and labeled with microbead-tagged anti-mouse IgM, and separated by MACS. The bound cells were then eluted, yielding a highly enriched population of $\mathrm{A} 2 \mathrm{~B} 5{ }^{+}$cells. After sorting, the cells were maintained for $1-2 \mathrm{~d}$ in DMEM/F12/N1 with $10 \mathrm{ng} / \mathrm{ml} \mathrm{bFGF}$ and $20 \mathrm{ng} / \mathrm{ml}$ PDGF-AA.

Transplantation. Myelin-deficient, immunodeficient shiverer $\left(\mathrm{MBP}^{\text {shishi }}\right) \times$ rag $2^{-1-}$ mice were generated as previously described (Windrem et al., 2008). Shiverer $\times$ rag $2^{-1-}$ and myelin wild-type rag $2^{-1-}$ newborn pups of either sex were both transplanted within a day of birth, using a total of 300,000 cells dispersed over five injection sites, also as described previously (Windrem et al., 2008). The myelin wild-type mice were killed for histology at $3,4.5,6,8$, or 12 months of age ( $n=3$ per time-point; 15 total), whereas engrafted shiverer brains were analyzed at $3,4.5,6,8$, or 12 months ( $n=3$ per time-point, except single mice at 6 and 8 months; 11 total). As allograft controls, EGFP $P^{+}$mouse GPCs were prepared and sorted as above and transplanted into rag2 $2^{-1-}(n=22)$ or shiverer $\times$ rag $2^{-/-}(n=8)$ mice on $\mathrm{P} 1$, at 300,000 cells/mouse in 5 sites, using the same procedure as that for hGPC xenografts. The A2B5-sorted mouse GPCs (mGPCs) were cultured for a week in DMEM/F12/N1 with 5\% FBS and then immunostained for the neuron-specific protein MAP2AB, so as to assess the incidence of neurons in this pool. Among 14 cultures derived from two separate sorts, an average of $2.1 \pm 0.5 \%$ expressed MAP2AB, consistent with the minor incidence of neuronal contaminants in these GPC sorts, as we had previously reported for A2B $5^{+} / \mathrm{PSA}-\mathrm{NCAM}$-based isolation of hGPCs (Windrem et al., 2004).

Immunolabeling. Mice were given barbiturate anesthesia, perfusion fixed with HBSS followed by $4 \%$ PFA. Brains were removed and postfixed for $2 \mathrm{~h}$ in cold PFA. Brains were cryopreserved in $6 \%$ and $30 \%$ sucrose (w/v), embedded sagittally in OCT, and cryosectioned at $20 \mu \mathrm{m}$. Cells were labeled with antibodies as listed in Table 1.

$B r d U$ tagging. To estimate the mitotic indices of each donor-derived phenotype at the time of death, the thymidine analog BrdU $(150 \mathrm{mg} / \mathrm{kg}$, i.p.) was given once daily for 5 consecutive days in the terminal week, and 
SHIVERER/RAG2 ${ }^{-1-}$ HOST MOUSE PROGENITORS HUMAN PROGENITORS
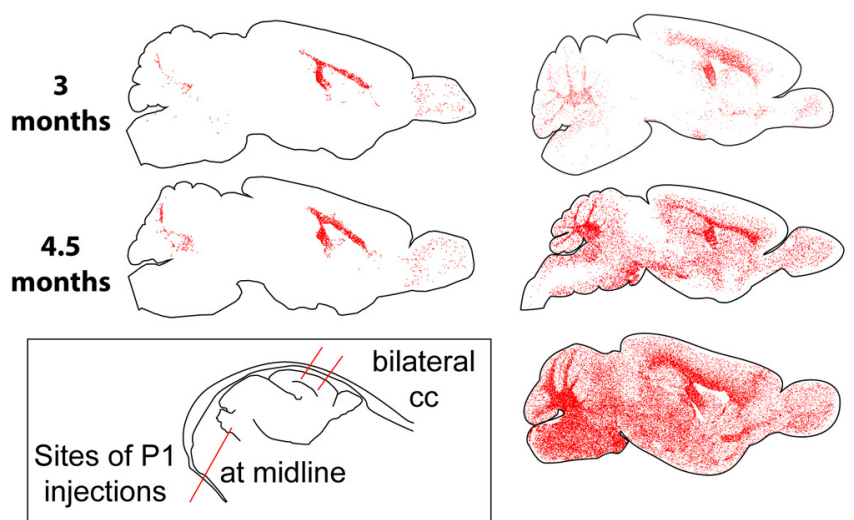

MOUSE PROGENITORS
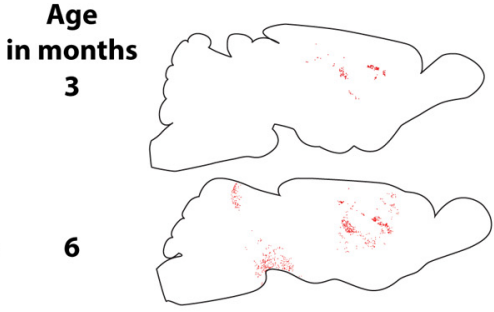

12

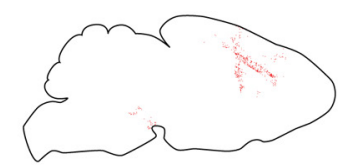

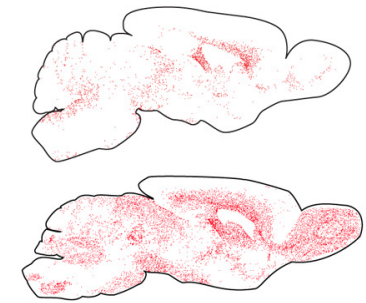

HUMAN PROGENITORS

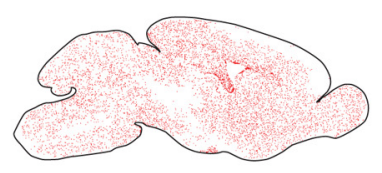

Figure 1. hGPCs colonize both wild-type and myelin-deficient immunodeficient host brain. hGPCs neonatally transplanted into either congenitally hypomyelinated shiverer $\times$ rag $2^{-/-}$mice (left columns) or normally myelinated rag $2^{-1-}$ mice (right columns) disperse and expand broadly throughout the brain as a function of age, and do so more aggressively than allografted mouse GPCs. hGPCs reach higher density in white matter than gray matter of the hypomyelinated shiverer, in contrast to their relatively uniform distribution in normally myelinated brain (right). Same-species neonatal allografts of EGFP-expressing mouse GPCs migrate and expand substantially less. Red dots indicate individual donor GPCs, as labeled by human nuclear antigen (human GPCs) or anti-GFP (mouse GPCs). Cells were mapped in $20 \mu \mathrm{m}$ sections using Stereo Investigator. Inset, Bottom left, Sites of neonatal injection, given anteriorly and posteriorly into the corpus callosum bilaterally, and as a single injection into the cerebellar peduncle.

the mice killed $2 \mathrm{~d}$ later so as to allow sufficient time for phenotypespecific antigenic expression. In BrdU-labeled samples, PDGFR $\alpha$ was used instead of NG2 to identify GPCs because NG2 proved incompatible with our BrdU labeling protocol. In control sections, we observed complete overlap of the NG2 and PDGFR $\alpha$-immunoreactive populations, except for a minor pool of morphologically apparent $\mathrm{NG}_{2}{ }^{+}$pericytes that did not express PDGFR $\alpha$.

Transplant mapping, cell counts, and phenotypic analysis. Montages for whole-section mapping of donor cells were generated on a Leica DM6000B equipped with a Leica DFC360FX high-speed camera system, using the HCX PL FLUOTAR $20 \times$ objective.

Quantification of callosal engraftment and donor cell phenotype in mice younger than 1 year were based on counts of the corpus callosum in three equally spaced sections of each mouse. In the 1-year-old mice whose brains had substantial donor cell engraftment, quantification of donor cell phenotypes in the corpus callosum was performed stereologically, by optical fractionator (West, 1999). We used a stereology system (StereoInvestigator; MicroBrightField), consisting of an Olympus BX-51 microscope equipped with a Ludl motorized stage, Heidenhain $z$-axis encoder, and Optronics QuantiFire video camera. Within each corpus callosum, from a random starting point, six sections equidistantly spaced $576 \mu \mathrm{m}$ apart were selected for analysis. After outlining the boundaries of the corpus callosum and establishing upper and lower exclusion zones of $10 \%$ of section thickness, a set of counting frames was placed by the software in a systematic random fashion to cover the corpus callosum of each section at $\sim 40$ sites. At each sampling site, the system acquired photographs at $1 \mu \mathrm{m}$ intervals along the $z$-axis through the sample rectangular prism $(x y z=80 \mu \mathrm{m} \times 80 \mu \mathrm{m} \times 16 \mu \mathrm{m})$. Photographs were taken at $400 \times$ or higher magnification. Cells were counted in the optical section in which they first came into focus.

\section{Results}

Neonatally engrafted hGPCs progressively expand in the murine forebrain

Using both homozygous shiverer ( $\left.\mathrm{MBP}^{\text {shi/shi }}\right)$ and myelin wildtype mice, each crossed to rag $2^{-/-}$immunodeficients, we evaluated the absolute numbers, relative proportions, and geographic distributions of human donor cells in neonatally engrafted recipients. In both cases, to generate mice chimeric for hGPCs, we transplanted newborn mouse pups with hGPCs isolated from second-trimester fetal human brain tissue, using immunomag- netic isolation of the $\mathrm{A} 2 \mathrm{~B}^{+}{ }^{+} / \mathrm{PSA}-\mathrm{NCAM}^{-}$phenotype. The cells were then delivered to the test mice using a five site intracerebral injection protocol that targeted the corpus callosum and cerebellar peduncle, as we have previously described (Windrem et al., 2008). As graft hosts, we used either newborn rag2 $2^{-/-}$immunodeficient myelin wild-type pups or hypomyelinated homozygous shiverer $\times \mathrm{rag}^{-1-}$ pups. Each mouse was transplanted with 300,000 GPCs delivered at five forebrain sites (Windrem et al., 2008), either with xenografted hGPCs, or as allograft controls, with $\mathrm{EGFP}^{+}$mGPCs, isolated via A2B5-based sorting from the cortices of P1 EGFP knock-in transgenic mice. The hGPCengrafted myelin wild-type mice were assessed for histology at 3 , $4.5,6,8$, or 12 months of age, whereas engrafted shiverer brains were analyzed at $3,4.5,6,8$, or 12 months (generally $n=3$ mice/time-point/genotype).

In the first 3 months following transplantation, hGPCs migrated widely to progressively engraft the forebrain white matter tracts (Fig. 1). During this period, the distribution of hGPCs in hypomyelinated and normally myelinated mouse brain were analogous. By 4.5 months of age, the dispersal pattern of hGPCs in wild-type mice was noted to differ from that in hypomyelinated shiverers, in that whereas hGPCs infiltrated in a relatively uniform fashion in both the gray as well as the white matter in myelin wild-types, hGPCs transplanted into shiverer mice preferentially expanded in the callosal and capsular white matter, in which they gave rise to new oligodendrocytes as well as additional GPCs and astrocytes (Fig. 1). Nonetheless, in both surviving shiverers and myelin wild-types, infiltration of the cortical and subcortical gray by migrating hGPCs ensued such that, by 1 year of age, donor hGPCs were distributed in a relatively uniform manner throughout both the white and gray matter.

In contrast to the aggressive expansion of xenografted hGPCs, $\mathrm{EGFP}^{+}$mouse GPCs allografted into myelin wild-type rag1 ${ }^{-/-}$ mice dispersed but did not expand substantially over time, nor did they migrate substantially beyond white matter tracts (Figs. 1 and 2A). Nonetheless, those EGFP-identified mGPCs allografted into shiverer brains did indeed expand as $\mathrm{NG}^{+}$progenitors within the hypomyelinated white matter (Fig. 2C,D, G), matur- 
ing therein into transferrin- and $\mathrm{MBP}-$ expressing oligodendroglia (Fig. 2E, F,H) and ultimately forming mature myelin (Fig. 2B), just as did hGPCs delivered into shiverer. However, allografted mGPCs manifested little dispersal beyond the major white matter tracts, compared with the widespread dispersal of hGPCs in shiverer as well as myelin wild-type hosts (Figs. 1 and $2 A$ ). Thus, the hypomyelinated shiverer brain could be myelinated by allografted murine as well as by xenografted hGPCs, but only hGPCs manifested preferential expansion and dominant colonization of the murine subcortical and neocortical gray matter.

\section{hGPCs actively excluded resident murine glial progenitors}

To define the dynamics of hGPC dispersal in the mouse brain, we used speciesspecific antibodies against the glial progenitor proteoglycan NG2 to map the respective locations of human donor and murine host $\mathrm{NG}_{2}{ }^{+}$cells, as a function of time after neonatal transplant. Analyzing 300 - $\mu \mathrm{m}$-wide columns of callosal wall extending from the lateral ventricle to the pial surface, we noted a progressive expansion of the hGPC pool relative to that of the host, with a serially expanding border between the two at all time-points. In both myelin wild-type and shiverer hosts, we noted that by 3 months of age, human $\mathrm{NG}^{+}{ }^{+}$cells typically replaced mouse $\mathrm{NG}^{+}{ }^{+}$cells in the corpus callosum (Fig. $3 A, B$ ). At that relatively early time-point, donor progenitors begin to advance into the lower layers of cortex, whereas more superficial cortical layers remain inhabited principally by murine $\mathrm{NG}^{+}{ }^{+}$GPCs (Fig. $3 C$ ). By 8 months, the human hGPCs have invaded the superficial cortex, whereas endogenous murine GPCs have become sequestered in the most superficial cortical layers (Fig. 3D). This process of hGPC expansion in the host forebrains continued, such that, by 1 year of age, the murine progenitors were largely replaced by transplanted human cells, often completely so (Fig. 2 E, F). Indeed, in 3 of 4 animals assessed at 1 year, no remaining mouse GPCs could be identified in the sampled forebrain sections. The geographical advance of donor progenitor cells is typically characterized by a discrete advancing front, which demarcates their border with host murine cells (Fig. $3 C-E$ ). Remarkably, isolated GPCs of either species were rarely noted behind these borders, suggesting the potency of the repulsive interactions likely characterizing the relationship of these analogous but heterospecific phenotypes.

The concurrent expansion of human cells from the callosum and elimination of endogenous progenitors proceeded with approximately exponential decay kinetics, and did so in both the cortical and subcortical gray matter, with striatal and basal forebrain infiltration by hGPCs occurring concurrently with neocortical invasion. Importantly, while the geographic patterns and timing of GPC migration proved analogous in both hypomyelinated shiverer and myelin wild-type hosts (Fig. 1), the relative degrees of local intracompartmental expansion differed, in that hGPCs expanded in the callosal environment of shiverer mice to a notably greater extent than their myelin wild-type counterparts (compare callosal hGPC densities between shiverer and wild-type hosts in Fig. 1). These observations suggested that the dominant colonization of the mouse brain by hGPCs reflected not only a species-selective competitive advantage of human over mouse GPCs, but also a context-dependent instruction of relative expansion and phenotypic differentiation.

\section{hGPCs differentiate in a context-dependent fashion}

On that basis, we next sought to assess the responsiveness of engrafted hGPCs to the host environment, by comparing their phenotypic differentiation in congenitally hypomyelinated and normally myelinated murine recipients. To that end, we used stereological analysis of progenitor-derived human astrocytes and oligodendrocytes to define the relative representation of each phenotype in the corpus callosum of neonatally xenografted shiverer and wild-type mice. In one year-old shiverer recipients, $>40 \%$ of human cells in the callosum expressed the oligodendrocytic protein CNP. In contrast, $<10 \%$ of human callosal cells in myelin wild-type mice did so at that point $(p<0.001$ by 2 -way ANOVA with Bonferroni $t$ test; Fig. $4 A$ ). Accordingly, $>50 \%$ of the human donor-derived cells remained as glial progenitors in the myelin wild-type chimeras, while $<15 \%$ do in the shiverer $(p<0.001$, Bonferroni $t$ test). Yet in contrast to the marked difference in oligodendrocytic differentiation by hGPCs between shiverer and wild-type mice, the relative proportions of engrafted human cells that developed $\mathrm{GFAP}^{+}$astrocytic phenotype proved no different in the two recipient models (Fig. 4A). 

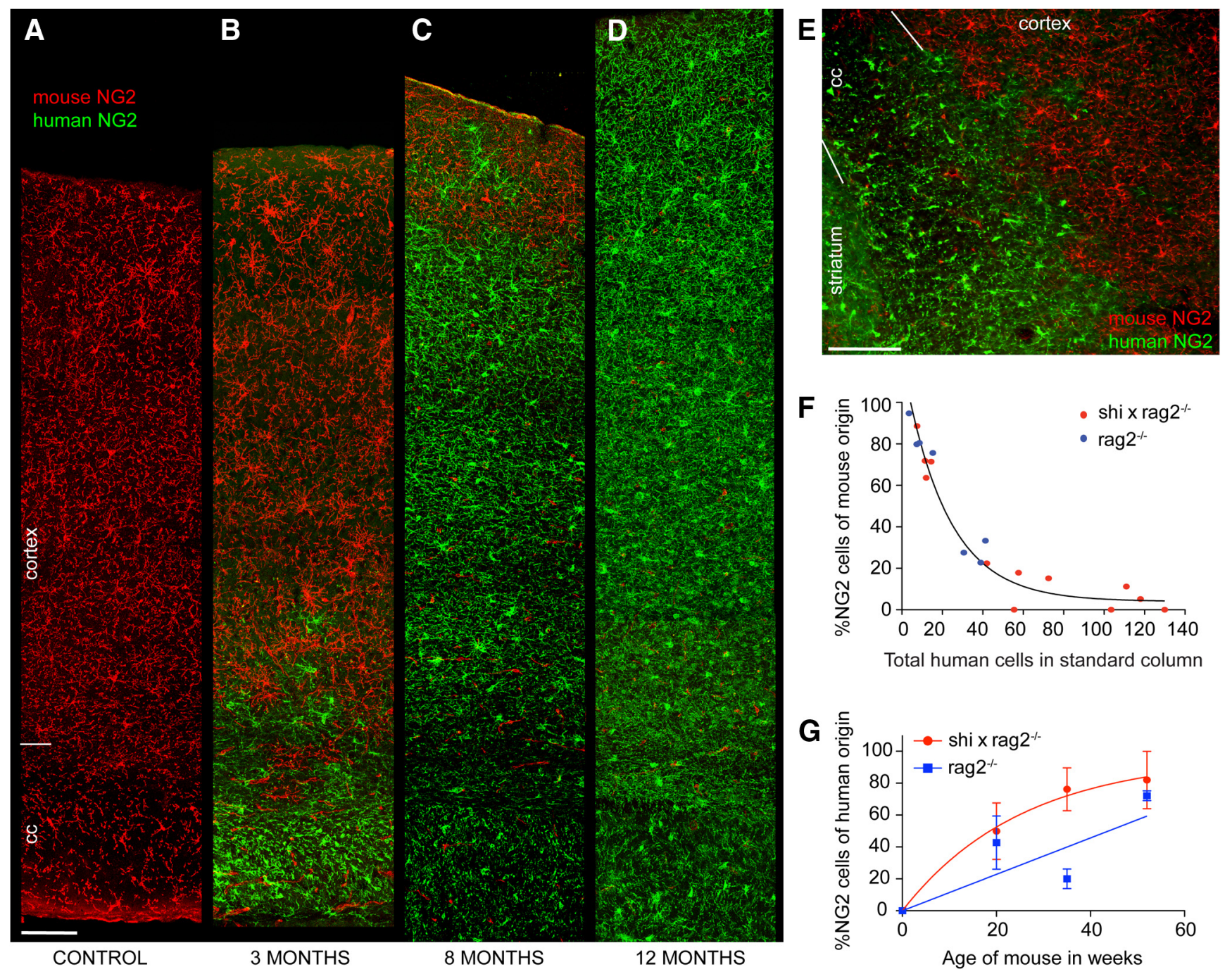

Total human cells in standard column

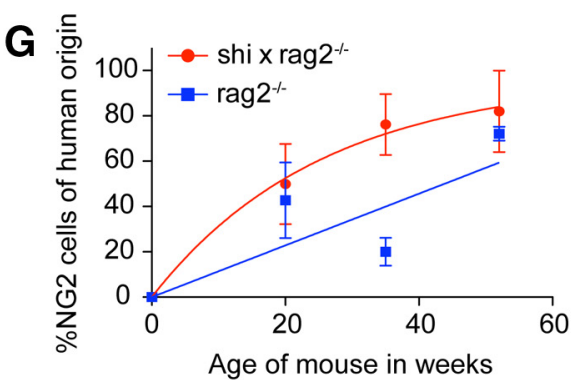

Figure 3. Progressive domination of murine forebrain by human glial progenitors. $\mathrm{hGPC}$ replacement of mouse glial progenitor cells (mGPCs) in both shiverer and normally myelinated rag ${ }^{-1-}$ mice, visualized using species-specific antibodies to the GPC chondroitin sulfate proteoglycan NG2. A, An unengrafted control showing the normal distribution of mouse NG2 ${ }^{+}$cells (red), spanning the distance from the ventricular ependyma to the pial surface; 3 months of age. $\boldsymbol{B}-\boldsymbol{D}$, Analogous radial strips spanning the ventricular to the pial surface, including the corpus callosum and cortex, of shiverer $\times$ rag2 ${ }^{-1-}$ mice engrafted neonatally with hGPCs. These images, taken at $3(\boldsymbol{B}), 8(\boldsymbol{C})$, and $12(\boldsymbol{D})$ months of age, show the systematic expansion of hNG2-defined hGPCs from the callosum to the cortical mantle, and the concurrent displacement of endogenous murine NG2 ${ }^{+}$cells. Red represents mouse NG2; green represents human NG2.E, Higher-magnification image of the mGPC-hGPC border in a 3-month-old myelin wild-type rag $2^{-1-}$ mouse demonstrates the sharp demarcation between the advancing human and retreating mouse glial progenitors. In myelin wild-type mice, by this time-point, hGPCs have replaced mGPCs throughout the corpus callosum, the hippocampus, and lower layers of cortex; mouse NG2 ${ }^{+}$cells still dominate the superficial layers of the cortex. $\boldsymbol{F}$, The replacement of host mouse cells by transplanted hGPCs in both shiverer/rag $2^{-I-}$ (red dots) and normally myelinated rag ${ }^{-1-}$ (blue dots) mouse callosal-cortical strips, plotted as the percentage of mouse NG2 ${ }^{+}$cells versus the number of human NG2 ${ }^{+}$cells/radial column. Across all time-points, as the total number of human cells in a radial column increased, the proportion of host mouse GPCs fell. G, The kinetics of hGPC replacement of endogenous mGPCs, as a function of time, in both shiverer and myelin wild-type $\times$ rag 2 null hosts. In both cases, colonization by hGPCs eventually yielded the substantial replacement and, in some cases, the apparent elimination of the endogenous mouse progenitor population. Scale bars: $\boldsymbol{A}-\boldsymbol{E}, 100 \mu \mathrm{m}$.

In absolute numbers, stereological estimation revealed that many times more human cells engrafted into the callosa of shiverer than in myelin wild-type mice (2-way ANOVA, $p<0.0001$ ) (Fig. 4B). In particular, the numbers of donor-derived oligodendrocytes and progenitors were each significantly higher in the shiverer than the myelin wild-type brains ( $p<0.001$ and $<0.01$, respectively, by Bonferroni $t$ tests), suggesting that the hypomyelinated environment encouraged selective expansion of donor hGPCs, as well as facilitating their oligodendrocytic differentiation (Fig. 4B).

It is important to note that the selective expansion of donor hGPCs in the shiverer brains appeared to be a product of the hypomyelinated environment, and not of xenograft per se, because neither the number nor relative proportion of hGPCs in neonatally xenografted wild-type callosa differed from the corresponding numbers of mouse progenitors in untransplanted wild- type controls (Fig. 4C,D). At baseline, $3.5 \pm 0.2 \%$ of callosal cells in untransplanted 1-year-old rag2-null mice were identified as GPCs by their expression of mouse NG2. Yet when 1-year-old hGPC engrafted rag2-nulls were similarly evaluated, using species-specific anti-NG2 antibodies, $3.6 \pm 0.3 \%$ of callosal cells were found to express human NG2. Similarly, in xenografted shiverers that survived to 1 year by virtue of neonatal transplantation, $4.1 \pm 0.2 \%$ of all callosal cells expressed human NG2 (Fig. $4 D$ ). As noted previously, by this late time-point, few, if any, mouse NG2 cells were noted to persist in the xenografted callosum, whether of shiverer or myelin wild-type hosts (Fig. $3 F, G$ ). In each case, the net density of parenchymal GPCs was unchanged despite the complete or near-complete replacement of mGPCs by hGPCs; by 1 year of age, 3\%-4\% of all cells expressed NG2, regardless of species or host genotype. 
In a similar vein, we noted that the proportion of human astrocytes increased as a function of time in the corpus callosum of the shiverer mice and was matched by a corresponding decrease in the proportion of mouse astrocytes (Fig. $4 E$ ); the net density of astrocytes was approximately preserved, despite the slow replacement of mouse by human astroglia. As with host colonization by hGPCs, we found that xenograft influenced only the species of resident astroglia and not their overall density. Together, these data indicated the context-dependent expansion and differentiation of hGPCs, with the speciesindependent preservation of phenotypespecific callosal cell densities for GPCs and astroglia alike.

\section{hGPCs remain mitotically active longer than allografted mGPCs}

We next asked whether species-selective differences in proliferative activity might contribute to the relative expansion of hGPCs. To that end, we treated matched cohorts of human and allografted mouse glial chimeric myelin wild-type mice with the DNA replication marker BrdU for $5 \mathrm{~d}$ before death at either 4 or 8 months of age. Death was followed by immunohistochemistry for BrdU together with phenotype-selective markers, followed by estimation of the respective mitotic indices of human and mouse GPCs. At both 4 and 8 months of age, we noted that the mitotic fraction of human donor cells was significantly higher than that of allografted mouse GPCs (Fig. 5A) (two-way ANOVA, $p<0.0005)$. These data indicate that hGPCs remain mitotically active long after the mitotic expansion of allografted murine glial progenitors has slowed, just as the xenografted hGPCs remain mitotically active long after endogenous GPC expansion has abated (Windrem et al., 2008); this in turn suggests that the selective expansion of hGPCs may contribute, at least in part, to their dominance over time in xenografted mice.

On that basis, we next asked whether the difference in expansion between human and mouse glia might also reflect phenotype-selective differences in the mitotic rate of progenitorderived daughters, relative to those derived from resident mouse GPCs. To this end, we immunostained BrdU-labeled sections of neonatally xenografted rag2-null myelin wild-type mice for the glial progenitor protein $\operatorname{PDGFR} \alpha$, the astroglial filament GFAP, or oligodendrocytic transferrin. Of note, in this experiment, we immunostained for human PDGFR $\alpha$ rather than NG2 to identify hGPCs, and transferrin rather than CNP to recognize oligodendroglia, so as to permit concurrent staining for BrdU, the fixation conditions for which obscure both NG2/CPSG4 and CNP immunodetection. We validated that our PDGFR $\alpha$ and transferrin immunostaining protocols exclusively recognized NG2- and CNP-expressing cells, respectively, in hGPC-xenografted brains
B
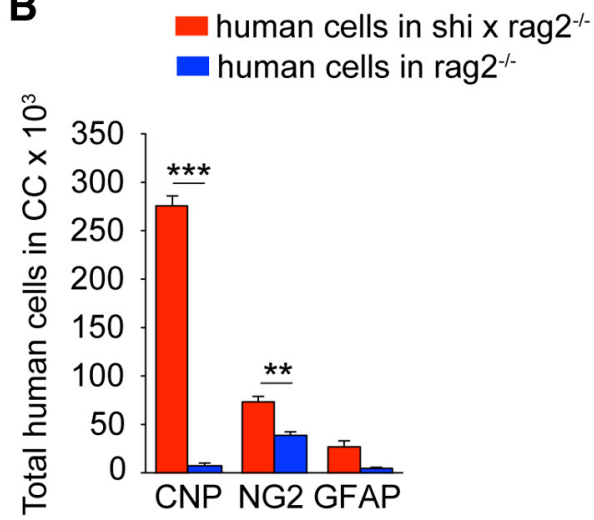

D

E

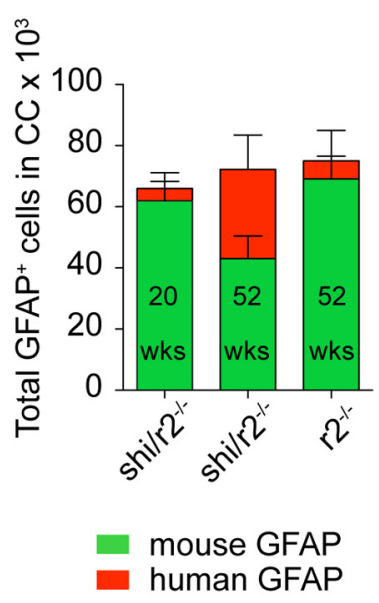

Figure 4. Phenotypic differentiation by engrafted hGPCs is context-dependent. $\boldsymbol{A}$, Differentiation at 1 year of hGPCs in the corpus callosum of congenitally hypomyelinated, transplant-rescued shiverer (red) and myelin wild-type mice (blue), each immunodeficient. In the shiverer callosum, $>40 \%$ of human donor cells had differentiated as CNP ${ }^{+}$oligodendrocytes; 作 corpus callosum, such that, by 1 year, almost half of all mouse callosal astrocytes have been replaced by hGPC-derived human astroglia, yielding white matter substantially chimeric for human astroglia as well as for hGPCs. ${ }^{* *} p<0.001$ (ANOVA with Bonferroni $t$ tests). ${ }^{* * *} p<0.0001$ (ANOVA with Bonferroni $t$ tests).

(data not shown). We found that, at both 4 and 8 months, the GPC fraction of all donor-derived cells remained significantly higher in hGPC-engrafted mice than in mice allografted with mGPCs (Fig. $5 B$ ), consistent with the higher sustained mitotic index of the human donor pool (Fig. 5A). Among GPC daughter cells, though, the production of oligodendroglia in myelin wildtype mice was more pronounced by murine than hGPCs at both 4 and 8 months (Fig. 5C), suggesting that allografted mouse GPCs are more biased toward rapid oligodendroglial differentiation than their human counterparts. In contrast, hGPCs xenografted into myelin wild-types appeared more biased to astrocytic differentiation, with evident donor-derived astrocytic accumulation between 4 and 8 months (Fig. 5D). Accordingly, the mitotic index of donor-derived astroglia was significantly higher in human than in allografted murine GPCs (Fig. 5E). Indeed, although human astrocytes typically comprised $5 \%-10 \%$ of BrdU ${ }^{+}$cells in wild-type recipients, no $\mathrm{BrdU}^{+}$astrocytes were identified in 
A
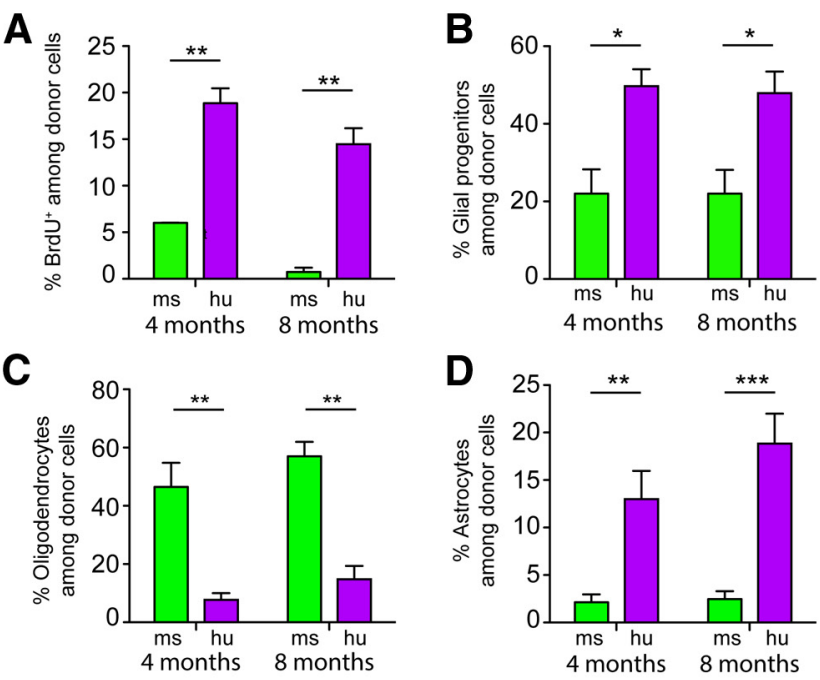

E
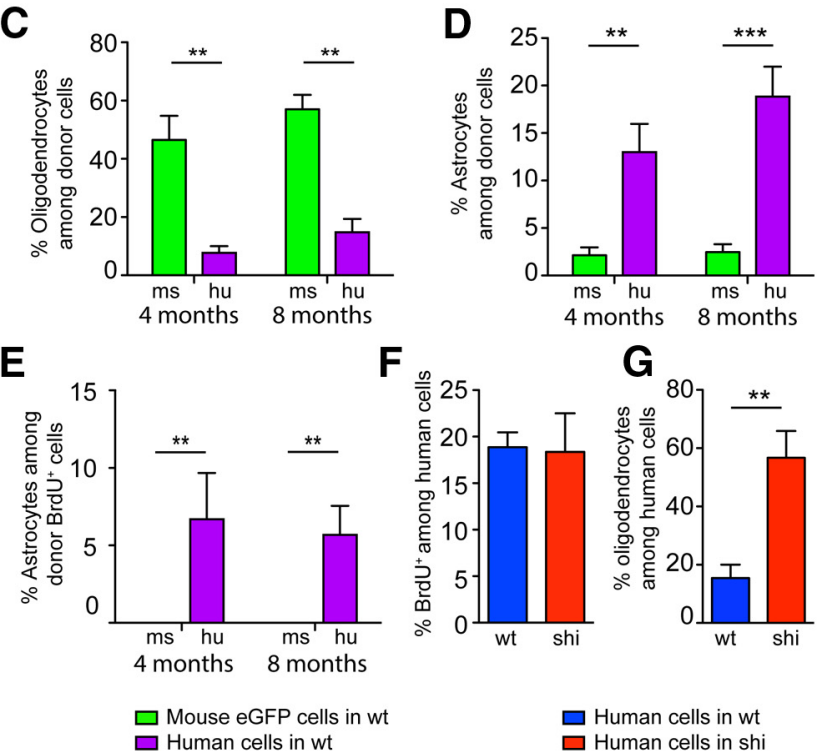

$\mathbf{F}_{\frac{0}{2}}$

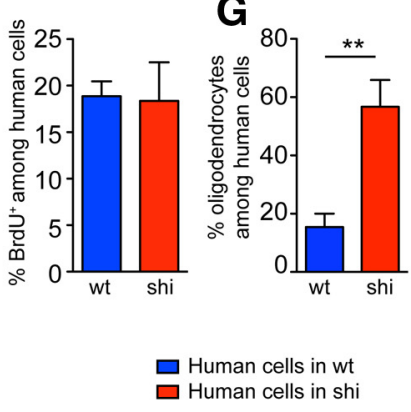

Figure 5. hGPCs expand preferentially relative to mouse GPCs in the murine environment. Neonatal chimeras were established in myelin wild-type $\mathrm{rag}^{-1-}$ mice, with either human or allogeneic EGFP-tagged mouse GPCs. These were maintained until 4 or 8 months of age, then injected for $5 \mathrm{~d}$ preterminally with BrdU to label the mitotic fraction of resident cells. The mice were then killed and callosal BrdU-tagged cells immunophenotyped. $A$, At both 4 and 8 months, EGFP $^{+}$mGPCs (green bars) exhibited substantially lower mitotic indices than the xenografted hGPCs (purple). ${ }^{* *} p<0.0005$ (one-way ANOVA). B, At both 4 and 8 months, higher proportions of human than mouse donor cells could be identified as PDGFR $\alpha$-defined glial progenitor cells. C, In contrast, allografted mouse GPCs were significantly more likely to differentiate as transferrin-defined oligodendrocytes than were their human counterparts, when injected into matched myelin wild-type recipients. $\boldsymbol{D}$, Substantially higher proportions of human donor cells differentiated as GFAP-defined astrocytes, compared with allografted mouse GPCS. $\boldsymbol{E}$, Strikingly, the mitotic index of these astrocytes differed dramatically between mouse and human donors: No mouse $\mathrm{GFAP}^{+}$cells were found to incorporate BrdU at either 4 or 8 months, indicating that, although new human astrocytes were continuously added to these adult brains from resident $\mathrm{hGPCS}$, astrocytic recruitment from murine progenitors appeared to be nil. $\boldsymbol{F}$, At the 4 month time-point studied, the mitotic index of callosal hGPCs did not differ between shiverer and myelin wild-type recipients. $\mathbf{G}$, However, a higher percentage of hGPCs differentiated as transferrin ${ }^{+}$oligodendrocytes in shiverer than in normally myelinated mice, again highlighting the context-dependent differentiation of hGPCs following their widespread, seemingly cell-autonomous migration. ${ }^{*} p<0.01$ (ANOVA with Bonferronit test). ${ }^{* *} p<0.001$ (ANOVA with Bonferroni $t$ test). ${ }^{* *} p<0.0001$ (ANOVA with Bonferroni $t$ test).

mGPC-allografted brains (Fig. 5E). These data indicate that astrocytic replacement in myelin wild-type glial chimeras derived almost entirely from engrafted human progenitors and thus suggest that hGPC-engrafted mice may experience slow replacement of their astrocytic as well as their progenitor populations. In addition, these findings highlight the persistence of both GPC phenotype and mitotic potential among xenografted human cells as contributing factors for the competitive dominance of hGPCs in the chimeric brain environment.

We next asked whether the mitotic index of human donor GPCs and their derivatives differed whether they were transplanted into wild-type or hypomyelinated recipients. We found no difference in the mitotic index of human donor cells whether introduced to wild-type or shiverer brain (Fig. $5 F$ ) but did note that the hGPCs were significantly more likely to generate oligodendroglia in the shiverer callosum, relative to that of myelin wild-types (Fig. 5G). No such differences were seen in the callosal production of GPCs or astrocytes as a function of recipient environment (data not shown). These observations again point to the pronounced context dependence of phenotypic differentiation by xenografted hGPCs and are consistent with the preferential instruction of GPCs toward oligodendroglial lineage in the shiverer environment.

\section{Human astrocytes exhibit cell-autonomous maturation in chimeric mice}

To assess the species-determined features of glial differentiation in these hGPC chimeras, we also assessed the morphological phenotypes of their derived astrocytes, in myelin wild-type recipients. We assessed astrocytic morphologies, and domain architecture in particular, because human astroglia may be readily distinguished in vivo from those of mice, spanning considerably larger domain volumes and manifesting significantly greater fiber complexity (Oberheim et al., 2009). We found that, at 8-10 months of age, by which point the majority of all forebrain GPCs in these mice were of human origin, the recipient brains also exhibited large numbers and high relative proportions of human astrocytes, in both gray and white matter. As we had previously noted in an assessment of human astroglial contributions to cognition in glial chimeric mice, the engrafted human glia in murine chimeras appear to develop and mature in a cell-autonomous fashion, in that their diameter, domain size, and morphology all approximate that of astrocytes in the normal adult human brain (Han et al., 2013). In each environment, including both the xenografted mouse brain and native human brain, human astroglia proved significantly more complex and phenotypically heterogeneous than host murine astrocytes (Fig. 6). As we have noted, the replacement of host GPCs by hGPCs leads, over time, to the effective chimerization of the recipient mice with human astrocytes. Because these astrocytes evidently retain human-specific pleomorphism and domain complexity, the astrocytic humanization of these chimeric brains appears to give rise to hominidspecific glial architecture as well as physiology in the murine brain.

\section{Discussion}

In this study, we engrafted neonatal mice with hGPCs, so as to evaluate the dispersal, interspecific competitive interactions, and context-dependent fate determination of xenografted hGPCs. We found that a large proportion of glial cells within the recipient mice, often all GPCs and a large proportion of astrocytes, and oligodendrocytes as well, when using hypomyelinated hosts, were ultimately replaced by human donorderived cells. The extent of this colonization of the mouse brain by human glia appeared so robust that we quantitatively evaluated the absolute numbers, relative proportions, and geographic distributions of human donor cells in the neonatally engrafted recipients. This analysis revealed that hGPCs so dominated and enjoyed such a competitive advantage over their murine counterparts that, by 9 months in vivo, virtually all glial progenitors within these mouse brains were typically replaced by hGPCs (Fig. 1).

The higher mitotic index of human donor cells relative to resident mouse GPCs in the host brains suggested a basis for our prior observation that, in shiverer homozygotes, an initial dose of 
300,000 cells/recipient expanded by at least 40 -fold by 12 months, to an average of $12 \times 10^{6}$ human glia in those animals rescued by neonatal progenitor transplantation (Windrem et al., 2008). Our present observations extend this analysis substantially by demonstrating that, in both the shiverer homozygotes and myelin wild-type hosts, the selective expansion of the human donor glia appears in part a product of the more sustained proliferation of hGPCs. Because the human donor GPCs are derived from the late secondtrimester SVZ, they might be expected to divide for up to 9 months after isolation; our data suggest that they sustain elements of that cell-autonomous program of expansion in the mouse host.

Importantly, shiverer and wild-type recipient mice differed in the compositions of their donor-derived phenotypes after neonatal chimerization. Whereas in xenografted adult shiverers, virtually all surviving oligodendrocytes were of human donor origin, in wild-type recipients, few donor-derived oligodendrocytes were apparent; rather, these brains had an abundance of hGPCs and astrocytes, the relative proportions of which increased with age. In both recipient environments, the human glial progenitors typically outcompeted their murine counterparts. This process was dynamic in scope and overtly competitive, in that the hGPCs typically expanded outwards from their periventricular and callosal points of introduction, in advancing waves that appeared to repulse resident murine progenitors, which appeared to die concurrent with their replacement, both in situ and upon retreat to the cortical surface (Fig. 3). The hGPCs progressively expanded until achieving a relatively uniform distribution, in tissue densities not significantly different from those of the mouse GPCs that they replaced; their assumption of an asymptotic distribution appeared analogous to that reported developmentally by Bergles and colleagues (Hughes et al., 2013). The repulsive signals to which the murine GPCs responded are unknown, as is the molecular basis for the competitive dominance of the hGPCs. Several recent studies have identified differential expression of both MYC- and hedgehog-dependent pathways as contributing to clonal dominance during early ontogeny (Clavería et al., 2013; Amoyel and Bach, 2014; Amoyel et al., 2014), and we are now assessing differential gene expression by mouse and hGPCs in vivo in an attempt to define analogous regulators of competition that may distinguish murine and hGPCs. Yet regardless of their mechanistic basis, the competitive interactions between mouse and hGPCs yielded the slow but inexorable glial humanization of these brains, first by hGPCs, and then by their derived astrocytes, as resident mouse astrocytes underwent normal turnover in adulthood, with replacement from the now-humanized progenitor pools (Fig. 3).

The value of such humanized models of in vivo brain phenotypes and function is especially clear when one considers that human astrocytes have functional competencies unique to hominids. Human astrocytes are more numerous, larger, and more structurally complex than those of infraprimate mammals, raising the possibility that the functional roles of glia have expanded during evolution (Oberheim et al., 2006, 2009). These evolutionary changes are of particular interest because astrocytes have been shown to play vital roles in information processing within the CNS (Kang et al., 1998; Araque et al., 1999). Astrocytes are required for synaptogenesis and maintenance of synaptic density (Ullian et al., 2001), and a number of specific astrocytic modulators of synaptic plasticity have been identified, including the glypicans (Allen et al., 2012) and TNF $\alpha$ (Stellwagen and Malenka, 2006), among others. Importantly, these ligands may be differentially expressed by human astroglia, thus imparting differential potency to human astroglia in the regulation of synaptic plasticity, relative to infraprimate glia (Oberheim et al., 2009; Han et al., 2013). As a result, the greater structural complexity of human astrocytes relative to those of rodents is accompanied by functional differences: human astrocytes propagate $\mathrm{Ca}^{2+}$ wave significantly faster than rodents, and human glial chimeric mice exhibit both enhanced long-term potentiation and facilitated learning in a variety of conditioned response paradigms and cognitive tasks (Han et al., 2013). Together, these observations suggest that the species-specific structural complexity of human astrocytes endows these cells with a fundamentally greater functional importance to synaptic modulation than that of their infraprimate counterparts.

Human astrocytes may differ substantially from their murine counterparts in their origin as well as their function. In our chimeras preterminally tagged with the mitotic marker BrdU, we found that, although new human astrocytes were continuously added to these brains, as recruited from resident engrafted hGPCs, astrocytic production from murine progenitors appeared to be nil; no mouse $\mathrm{GFAP}^{+}$cells were found to incorporate BrdU at either 4 or 8 months (Fig. $5 E$ ). This observation may suggest a fundamental distinction in the origin of new astroglia in the brains of adult rodents and humans; although in mice resident astrocytes have been reported as the principal source of new astrocytes in adulthood (Ge et al., 2012), with GPCs serving prin- 
cipally as oligodendrocyte progenitors ( $\mathrm{Bu}$ et al., 2004; Nishiyama et al., 2009; Kang et al., 2010; Tripathi et al., 2010), human glial progenitors are notably bipotential for both oligodendrocytes and astrocytes in the adult brain (Nunes et al., 2003; Sim et al., 2009; Sim et al., 2011).

Our ability to generate mice in which the bulk of the glial population is of human origin opens the possibility of constructing human glial chimeras in a patient-specific and diseasedefined manner, using hGPCs derived from embryonic stem cells and induced pluripotential cells. In particular, the ability to generate astrocytes (Krencik et al., 2011), as well as glial progenitors and oligodendroglia (Wang et al., 2013), from human-induced pluripotential stem cells permits the construction of mice in which we may now assess the relative contributions of human glia to disease pathology in vivo. For instance, the derivation of hGPCs from pluripotential stem cells carrying the polyglutamine repeat expansion of Huntington disease (HD), and the establishment of human glial chimeras using those huntingtin mutant hGPCs, may permit us to define the specific contributions of glia to neuropathology in $\mathrm{HD}$, as well as to the clinical phenotype of HD. Similarly, the construction of human glial chimeras using hGPCs derived from induced pluripotential cells generated from patients with hereditary neuropsychiatric conditions may permit us to define and isolate the contribution of glia to these disorders, the phylogenetic appearance of which seems approximately concurrent with the evolution of morphological complexity by human astroglia (Horrobin, 1998; Oberheim et al., 2009). More broadly, such disease-specific induced pluripotential stem cellderived human glial chimeras may permit us to better define the role of glial dysfunction in a broad swath of nominally neuronal neurodegenerative and neuropsychiatric disorders, in which the relative contribution of glial pathology remains unclear and understudied.

\section{References}

Allen NJ, Bennett ML, Foo LC, Wang GX, Chakraborty C, Smith SJ, Barres BA (2012) Astrocyte glypicans 4 and 6 promote formation of excitatory synapses via GluA1 AMPA receptors. Nature 486:410-414. CrossRef Medline

Amoyel M, Bach EA (2014) Cell competition: how to eliminate your neighbours. Development 141:988-1000. CrossRef Medline

Amoyel M, Simons BD, Bach EA (2014) Neutral competition of stem cells is skewed by proliferative changes downstream of $\mathrm{Hh}$ and Hpo. EMBO J 33:2295-2313. CrossRef Medline

Araque A, Parpura V, Sanzgiri RP, Haydon PG (1999) Tripartite synapses: glia, the unacknowledged partner. Trends Neurosci 22:208-215. CrossRef Medline

Bu J, Banki A, Wu Q, Nishiyama A (2004) Increased NG2(+) glial cell proliferation and oligodendrocyte generation in the hypomyelinating mutant shiverer. Glia 48:51-63. CrossRef Medline

Clavería C, Giovinazzo G, Sierra R, Torres M (2013) Myc-driven endogenous cell competition in the early mammalian embryo. Nature 500:39-44. CrossRef Medline

Ge WP, Miyawaki A, Gage FH, Jan YN, Jan LY (2012) Local generation of glia is a major astrocyte source in postnatal cortex. Nature 484:376-380. CrossRef Medline

Goldman SA, Schanz S, Windrem MS (2008) Stem cell-based strategies for treating pediatric disorders of myelin. Hum Mol Genet 17:R76-R83. CrossRef Medline

Goldman SA, Nedergaard M, Windrem MS (2012) Glial progenitor cellbased treatment and modeling of neurological disease. Science 338:491495. CrossRef Medline

Han X, Chen M, Wang F, Windrem M, Wang S, Shanz S, Xu Q, Oberheim NA, Bekar L, Betstadt S, Silva AJ, Takano T, Goldman SA, Nedergaard M (2013) Forebrain engraftment by human glial progenitor cells enhances synaptic plasticity and learning in adult mice. Cell Stem Cell 12:342-353. CrossRef Medline

Horrobin DF (1998) Schizophrenia: the illness that made us human. Med Hypotheses 50:269-288. CrossRef Medline

Hughes EG, Kang SH, Fukaya M, Bergles DE (2013) Oligodendrocyte progenitors balance growth with self-repulsion to achieve homeostasis in the adult brain. Nat Neurosci 16:668-676. CrossRef Medline

Kang J, Jiang L, Goldman SA, Nedergaard M (1998) Astrocyte-mediated potentiation of inhibitory synaptic transmission. Nat Neurosci 1:683692. CrossRef Medline

Kang SH, Fukaya M, Yang JK, Rothstein JD, Bergles DE (2010) NG2+ CNS glial progenitors remain committed to the oligodendrocyte lineage in postnatal life and following neurodegeneration. Neuron 68:668-681. CrossRef Medline

Krencik R, Weick JP, Liu Y, Zhang ZJ, Zhang SC (2011) Specification of transplantable astroglial subtypes from human pluripotent stem cells. Nat Biotechnol 29:528-534. CrossRef Medline

Nishiyama A, Komitova M, Suzuki R, Zhu X (2009) Polydendrocytes (NG2 cells): multifunctional cells with lineage plasticity. Nat Rev Neurosci 10: 9-22. CrossRef Medline

Nunes MC, Roy NS, Keyoung HM, Goodman RR, McKhann G 2nd, Jiang L, Kang J, Nedergaard M, Goldman SA (2003) Identification and isolation of multipotential neural progenitor cells from the subcortical white matter of the adult human brain. Nat Med 9:439-447. CrossRef Medline

Oberheim NA, Takano T, Han X, He W, Lin JH, Wang F, Xu Q, Wyatt JD, Pilcher W, Ojemann JG, Ransom BR, Goldman SA, Nedergaard M (2009) Uniquely hominid features of adult human astrocytes. J Neurosci 29:3276-3287. CrossRef Medline

Oberheim N, Wang X, Goldman S, Nedergaard SA (2006) Astrocytic complexity distinguishes the human brain. Trends Neurosci 29:547-553. CrossRef Medline

Roy N, Wang S, Harrison-Restelli C, Benraiss A, Fraser R, Gravel M, Braun M, Goldman S (1999) Identification, isolation, and promoter-defined separation of mitotic oligodendrocyte progenitor cells from the adult human subcortical white matter. J Neurosci 19:9986-9995. Medline

Roy N, Wang S, Jiang L, Kang J, Benraiss A, Restelli C, Fraser R, Couldwell W, Kawaguchi A, Okano H, Nedergaard M, Goldman S (2000) In vitro neurogenesis by progenitor cells isolated from the adult human hippocampus. Nat Med 6:271-277. CrossRef Medline

Sim FJ, Windrem MS, Goldman SA (2009) Fate determination of adult human glial progenitor cells. Neuron Glia Biol 5:45-55. CrossRef Medline

Sim FJ, McClain CR, Schanz SJ, Protack TL, Windrem MS, Goldman SA (2011) CD140a identifies a population of highly myelinogenic, migration-competent and efficiently engrafting human oligodendrocyte progenitor cells. Nat Biotechnol 29:934-941. CrossRef Medline

Stellwagen D, Malenka RC (2006) Synaptic scaling mediated by glial TNFalpha. Nature 440:1054-1059. CrossRef Medline

Tripathi RB, Rivers LE, Young KM, Jamen F, Richardson WD (2010) NG2 glia generate new oligodendrocytes but few astrocytes in a murine experimental autoimmune encephalomyelitis model of demyelinating disease. J Neurosci 30:16383-16390. CrossRef Medline

Ullian EM, Sapperstein SK, Christopherson KS, Barres BA (2001) Control of synapse number by glia. Science 291:657-661. CrossRef Medline

Wang S, Bates J, Li X, Schanz S, Chandler-Militello D, Levine C, Maherali N, Studer L, Hochedlinger K, Windrem M, Goldman SA (2013) Human iPSC-derived oligodendrocyte progenitor cells can myelinate and rescue a mouse model of congenital hypomyelination. Cell Stem Cell 12:252-264. CrossRef Medline

West MJ (1998) Stereological methods for estimating the total number of neurons and synapses: issues of precision and bias. Trends Neurosci 22: 51-61. CrossRef Medline

Windrem MS, Nunes MC, Rashbaum WK, Schwartz TH, Goodman RA, McKhann G 2nd, Roy NS, Goldman SA (2004) Fetal and adult human oligodendrocyte progenitor cell isolates myelinate the congenitally dysmyelinated brain. Nat Med 10:93-97. CrossRef Medline

Windrem MS, Schanz SJ, Guo M, Tian GF, Washco V, Stanwood N, Rasband M, Roy NS, Nedergaard M, Havton LA, Wang S, Goldman SA (2008) Neonatal chimerization with human glial progenitor cells can both remyelinate and rescue the otherwise lethally hypomyelinated shiverer mouse. Cell Stem Cell 2:553-565. CrossRef Medline 